\title{
EVALUACIÓN DE LOS \\ ESTEREOTIPOS DE GÉNERO EN EDUCACIÓN INFANTIL
}

\author{
Blanca Rodríguez Fernández ${ }^{1}$
}

Universidad de Almería

\section{Resumen}

En los últimos años, se ha producido un aumento en la investigación relativa a los estereotipos de género, es decir, en la idea, imagen o noción inmutable que un grupo social tiene sobre otro grupo, al cual se le atribuyen como correctas y de forma generalizada unos rasgos distintivos o habilidades, diferenciando a un género de otro. Los estereotipos pueden ser diferentes dependiendo de la nacionalidad, etnia, clase socioeconómica, sexo, edad, profesión, etc., e influyen en los niños y niñas desde una edad temprana. Dicho interés, motivado por causas variadas como el aumento de la violencia de género o la mayor sensibilización acerca de la igualdad ha permitido tomar conciencia de la necesidad de continuar en la lucha para acabar con ellos.

Estos estereotipos culturalmente enraizados y transmitidos en los procesos de socialización tendrían una fuerte influencia en los/as niños/as ya desde edades preescolares. El presente estudio tiene como objetivo evaluar los estereotipos en 18 niños de 4 y 5 años (8 niños y 10 niñas) a través de un cuestionario breve de 12 ítems apoyado en tarjetas visuales, diseñado para el estudio. Más concretamente, se sondean cuestiones relativas a los estereotipos de género en lo relativo a aspecto físico, juguetes y profesiones. Los resultados confirman la existencia de estereotipos ya a edades tan tempranas, estableciendo patrones diferenciales entre sexos en lo relativo a colores, juguetes y profesiones. Desde nuestro papel como educadores, es crucial prestar atención a estos estereotipos para no continuar perpetuándolos y continuar trabajando con el objetivo de reducir las desigualdades entre géneros.

Palabras clave: estereotipo; género; igualdad de género; tipificación sexual.

\begin{abstract}
In recent years, there has been an increase in the investigation concerning gender stereotypes, that is the idea, image or immutable notion that a social group have about another group, to whom distinctive features or abilities are attributed as generally correct, by differentiating one gender from another. Stereotypes might be different depending on the nacionality, ethnic group, socioeconomic class, gender, age, profession, etc. and influence children since an early age. That interest, motivated by a variety of causes such as the increase in gender violence or the greater awareness of equality, has allowed us to become aware of the need to put an end to them.

These culturally rooted stereotypes and transmitted in socialization processes would have a strong influence on children since preschool age. The aim of the present study is to assess stereotypes in 18 four-and-fiveyear-old students ( 8 boys and 10 girls) through a brief 12 -item questionnaire supported by visual cards, especially designed fot the study. More precisely, we have probed issues related to gender stereotypes regarding physical appearance, toys and professions. The results verify the existence of stereotypes already at such early ages, by establishing differential patterns between the sexes regarding colors, toys and professions. From our role as educators, paying attention to these stereotypes is crucial so as not to continue perpetuating them and to continue working with the aim of reducing gender inequalities.
\end{abstract}

Key words: stereotype; gender; gender equality; sex-typing

\section{MARCO TEÓRICO}

\section{Introducción}

Durante los últimos años se ha producido un importante $y$, por otra parte, indispensable, interés en la investigación en el área de los estereotipos de género. Motivado en muchas ocasiones por la preocupación social que genera la desigualdad en este sentido, así como el aumento de los casos de la violencia de género, entre otras razones, lo cierto es que estos estereotipos son en muchas ocasiones herencia de ideas profundamente arraigadas en el acervo popular. Estas ideas y valores se trans-

Correspondencia: Blanca Rodríguez Fernández

Email: b_I_a_n_c_a_7@hotmail.com mitirían de generación en generación desde la infancia en el proceso de socialización y educación, siendo de este modo perpetuadas.

Sin ir más lejos, una de las razones por la cual es importante profundizar en los estereotipos en la infancia, es, que estos influyen directamente en la conducta de los niños a la hora de relacionarse con los demás. Por citar algunos ejemplos, podemos recurrir a la presencia de estereotipos para explicar por qué un niño no quiere vestir de rosa o una niña se niega a jugar al fútbol. Además, el apartarse de los estereotipos prefijados socialmente para cada género podría conducir a situaciones de marginación o incluso bullying. Esto sucede tanto en nuestro país como en el resto del mundo, pues, no se acepta al $100 \%$ a día de hoy que chicos tenga gustos o preferencias des-atípicas a las de un chico (o al revés). Sin embargo, también se ha mostrado que es posible revertir esta situación, siendo para ello fundamental la observación hacia los más pequeños, la 
educación en valores de igualdad y la comunicación fluida escuela-familia (Bosada, 2018). Además, estos estereotipos se interiorizan y asumen, teniendo considerables implicaciones en los adultos que serán el día de mañana. Por ello, es fundamental que, desde una edad temprana, los niños y niñas sepan valorar y respetar a las personas. Afortunadamente, cada vez contamos con más instrumentos para educar en igualdad desde las aulas, inculcando valores y trabajando en cooperación escuela, familia y sociedad.

Tal y como establecen Hernández Morales y Jaramillo Guijarro (2003), es importante no establecer separación escuela vs. familia, ya que, aunque se trate de contextos educativos distintos, ambos son cruciales en el desarrollo de los valores y de la moral del niño/a. En lo referido al ámbito del desarrollo afectivo-sexual, también es importante no descuidarlo. Debemos ser conscientes de la necesidad de saber respetar las diferencias y gustos de los demás, en este caso, los de la persona (niño/a) con el que nos comunicamos.

En términos comparativos, mujeres y varones tienen en común más similitudes que rasgos diferenciales. Sin embargo, hemos aprendido a representarnos mentalmente a unas y a otros como polos opuestos. Esta representación polarizada ha favorecido el desarrollo y fuerte arraigo de los estereotipos de género.

El presente trabajo es una primera aproximación a los estereotipos de género. En primer lugar, se presenta una introducción teórica en la que se precisan algunos conceptos clave, así como teorías sobre el proceso de transmisión de los estereotipos. En la parte empírica, se presentan los datos correspondientes a la evaluación realizada en niños/as preescolares, así como, el análisis y discusión de estos resultados y las implicaciones de los mismos.

Algunas precisiones terminológicas: sexo, género, estereotipo y rol de género $\mathrm{Al}$ abordar esta temática, en primer lugar, es fundamental precisar las diferencias existentes entre diferentes conceptos que si bien estrechamente relacionados, guardan importantes matices diferenciadores, y que en ocasiones se prestan a un mal uso o confusión en el habla cotidiana, como son el sexo, el género, identidad de género, rol de género, estereotipos y estereotipos, de género.

En primer lugar, el concepto de sexo se refiere a la cualidad biológica de las personas (Wallen, 2009). Hace referencia, por tanto, al aspecto más puramente fisiológico, clasificándose en dos (hombre/mujer), diferenciándose en los cromosomas, órganos reproductores, hormonas especificas o factores ambientales que también afectan a los fenotipos.

El concepto de género se encuentra inmerso dentro de lo cultural, ya que es el conjunto de características diferenciadas que la sociedad asigna a los individuos según su sexo, teniendo en cuenta comportamientos, atributos y cualidades que una sociedad ha creado y ha considerado perteneciente a un sexo u otro, es decir, más femeninos o masculinos. Por tanto, este concepto es dependiente de la cultura y el momento históricocultural. Cada sociedad, tendría un sistema sexo-género, con sus códigos y peculiaridades. Por ejemplo, una mujer puede nacer en China o en África, y a ambas se las identificará al nacer como mujeres, pero su cultura no espera de ellas los mismos comportamientos, roles o características, porque éstos comportamientos, las costumbres, las creencias, están definidas y diferenciadas por la cultura en la que viven.

Según Gallegos Argüello (2012), el concepto de identidad de género, hace referencia a cómo nosotros nos identificamos y expresamos ante la sociedad. Es nuestra conducta y actitud propia, la que elegimos y lo que nos identifica principalmente con el género al que pertenecemos.

En cuanto al término rol de género, se refiere a aquellas funciones y actividades que se supone que deben cumplir los individuos dependiendo del sexo al que pertenezcan. Por citar un ejemplo tradicionalmente perpetuado, a las mujeres se les atribuye el rol de cuidar de la familia mientras que el hombre es el encargado de sustentar a la familia en el ámbito económico. Esto es, el conjunto de normas sociales y comportamientos habitua- dos que son dirigidos como apropiados para los hombres y las mujeres en nuestra sociedad. El término estereotipo, procede del griego stereos (sólido) y typos (huella, impresión, molde), relacionando el significado con una imagen social preestablecida que se posee de un grupo, o de una persona en particular, y que, como su etimología indica, permanece resistente a modificaciones, aunque este si puede ser modificable, siendo comúnmente utilizado de forma peyorativa y/o despectiva. Cuando hablamos de estereotipos de género hacemos referencia al acuerdo generalizado por la sociedad, al pensamiento colectivo sobre lo que caracteriza a cada uno de los géneros GonzálezGavaldón (1999).

Por tanto, los estereotipos no son sino creencias (escrito en plural, pues rara vez suele existir una sola creencia), esquemas cognitivos acerca de los grupos, o miembros de dichos grupos que representarían nuestros conocimientos. Estas creencias son aplicables indistintamente para hacer referencia tanto a rasgos de personalidad, como a atributos físicos, conductas o roles y ocupaciones, condicionando y sesgando en gran medida la percepción, el recuerdo y la valoración de muchas de las características y conductas hacia los hombres y especialmente, hacia las mujeres. Así, por ejemplo, se asume en el acervo popular que "si eres mujer deberás llevar una determinada vestimenta o tendrás un determinado comportamiento o actitud en ciertas situaciones".

\section{La tipificación sexual y el papel de los estereotipos}

La tipificación sexual es la forma en la que socialmente se aprende a ser niño o niña. Comienza en el desarrollo cognitivo y evolutivo cuando se tiene entre 2 años y medio o 3 años según un estudio evolutivo (Shaffer, 2000). En ese momento, los niños y niñas ya comienzan a diferenciar, separar y catalogar los sexos, fijándose en caracteres externos, como, por ejemplo, la manera de vestir. En cualquier caso, entre los 3 y 5 años (la etapa de prescolar) los más pequeños creen que el hecho de sentirse niña o niño es algo fijo o estable imposible de cambiar y que subsistirá igual a lo largo de sus vidas. A esas edades, aún no alcanzan la madurez suficiente para ser conscientes de que lo único que es estable es el sexo (esto es, la parte biológica) y permanece a pesar de que las personas puedan cambiar de actividades y de apariencia o presentación (Kohlberg, 1969). Los demás cambios dependerán de la ropa que vistan, peinados u otros estereotipos o roles que los diferencian. Asimismo, es en la infancia, durante el proceso de socialización cuando comienzan a observarse, aprehenderse e interiorizarse la mayor parte de los estereotipos. En efecto, desde el nacimiento, se observan comportamientos diferenciales en el trato hacia los dos sexos. Sin ir más lejos, la primera pregunta que se realizan tantos los padres cómo los más allegados a la bienvenida de un bebé al mundo es saber cuál va a ser su sexo (EspinosaBayal, 2006). Desde el momento que lo saben, ya comenzarán a preparar unos juguetes u otros, ropa de un color u otro (azul para niño y rosa para niña, cómo normalmente se conoce), etc. es decir, antes de llegar al mundo, los niños y niñas ya tienen preparados unas pautas de cómo comportarse o con qué debe jugar. El hecho de tener unas expectativas para el bebé, va a tener unas consecuencias en su futuro: la posición en la que se sitúa el sexo femenino es de inferioridad en la sociedad actual (aunque afortunadamente, se está empezando a trabajar para igualar esta situación).

Del mismo modo, se establecen rasgos diferenciales en el tipo de personalidad y carácter socialmente aceptado. Así, el carácter masculino según el estudio realizado por Conway, Bourque y Scott (1996), se asocia con adjetivos como agresivo o infiel. Por el contrario, el sexo femenino se ha catalogado como dependiente, de carácter más dócil, expresivo y servicial. Rasgos como sensibles, cálidas, dependientes y orientadas hacia la gente se han empleado tradicionalmente para describir a las mujeres, con las consiguientes consecuencias que la interiorización de estos adjetivos puede conllevar a un nivel psicológico negati- 
vo. (González, 1999).

En un estudio clásico, Best (1985), realizó un estudio en 110 culturas no industrializadas examinando los roles asumidos por hombre y mujeres. Los resultados revelan que el conjunto de sociedades coincide en atribuir unos rasgos al sexo femenino y otros al sexo masculino. Así, ellas son quienes se ocupan de la crianza y educación, y son presionadas para ser más obedientes y más responsables. Los niños por su parte, son presionados para que se ocupen de lo laboral. Además, estos últimos presentaban más confianza en ellos mismos. Según Unger (1989), entre los 4 y 10 años los niños van tomando conciencia de qué se espera de ellos y van conformando su conducta a esas expectativas. Este autor, también nos dice que los padres que pertenecen a una clase social baja imitan con mayor fidelidad los papeles tradicionales masculinos y femeninos que se vienen dando desde el pasado y se los imponen con mayor exigencia a sus hijos. Ellos deben ajustarse al rol que la sociedad tiene respecto al sexo masculino, lo que conlleva a no mostrarse débil o ser menos sensibles, más fuertes, más dominantes, etc. (Silvestre Cabrera, 2010). Igualmente, estos estereotipos y roles están presentes, a veces de manera inconsciente en las aulas. Según Alonso Feijoo (2015), todos los disfraces infantiles en épocas de festividad como, por ejemplo, en carnaval, los disfraces de las niñas priman la belleza (en ocasiones incluso se les maquillan), mientras que los disfraces de los niños son mucho más profesionales, más serios, sin tener tan presente la belleza.

\section{Juguetes estereotipados}

Por otro lado, en el campo de la educación el juego tiene un papel importante, ya que se trata de una herramienta primordial para el aprendizaje y la socialización, ya desde una edad temprana. Los juegos son un reflejo del mundo, de la cultura y de la sociedad que nos rodea, por lo que a través de él se aprenden valores, roles, etc. (Erikson, 1974). Todo lo anteriormente señalado, condiciona enormemente cómo se actúa conforme a estos estereotipos: las niñas deberán jugar a juegos de servir y cuidar a los demás (las cocinitas, las mamás), mientras que los niños son alientados a tomar parte de a juegos en los que se demuestre su "hombría", expresen su carácter dominante, o sean deportes etiquetados tradicionalmente como de "hombres" (luchas, el fútbol...). En otras palabras, los juguetes de "niños" fomentan la curiosidad y la aventura mientras que los juguetes de "niñas" fomentan el lado sensible y maternal con muñecas (Vázquez, 2020). Esto acaba siendo interiorizado y asumido y supone un importante condicionante a la hora de escoger un juguete. Es por ello, por lo que para aprovechar al máximo las potencialidades que el juego nos brinda, los juguetes deben estar adaptados a cada edad, pero también, a los objetivos que se pretenden conseguir, priorizando los gustos y preferencias de los niños, antes que catalogar el juguete que se le quiere comprar atendiendo a estereotipos culturales, que no harán sino transmitir estas creencias erróneas. Es el sexo masculino quien sufre una mayor coacción a la hora de mostrar sus gustos o preferencias cuando se apartan de los estereotipos socialmente establecidos (Torres, 2018). Por citar algunos ejemplos y aunque afortunadamente esto está cambiando, los padres toleran mejor que una niña juegue con coches a que un niño juegue con muñecas (Lumbreras-Castellanos, 2013). Esto además constituye un fenómeno bastante extendido a nivel mundial independientemente del nivel de riqueza o clase social al que pertenezcan los niños y niñas. En efecto, es a día de hoy cuando más separación de juguetes según el género existe en relación a 50 años atrás. Sweet (2018), mostró que, mientras en 1975 sólo el 2\% de los juguetes estaban explícitamente catalogados para "niños" o para "niñas" en el catálogo de Sears (el catálogo más importante de juguetes de la época en los Estados Unidos), hoy en día todos los juguetes de la página web de Disney están divididos de esa forma. Sin embargo, también se ha mostrado que es posible revertir esta situación, siendo para ello fundamental la observación hacia los más pequeños, la educación en valores de igualdad y la comunicación fluida escuela-familia (Bosada, 2018)

\section{Igualdad de género}

La igualdad de género es el respeto y conocimiento de que todas las personas, sin importar el sexo al que pertenezca. Si bien, desde un marco legal la igualdad está amparada (Ley 2/2016, de 29 de marzo, de Identidad y Expresión de Género e Igualdad Social y no Discriminación-BOE) desgraciadamente a efectos prácticos encontramos grandes carencias que convierten esta igualdad en una utopía. A nivel global, las diferencias entre mujeres y hombres, llegan a ser considerables en las áreas más importantes de la vida de cualquier persona, como es en el ámbito social, económico y social. Por citar ejemplos más concretos, actualmente, cerca de 130 millones de niñas de todo el mundo no van a la escuela (BBC, 2017), la participación de las mujeres en el ámbito laboral no es igual de activa que la del sexo masculino, o incluso desempeñando un mismo trabajo, el salario de la mujer es inferior al del hombre (Gómez, 2018).

Esta desigualdad se manifiesta igualmente en el ámbito doméstico. Según señalan Chinchilla, Poelmans y León (2005), en España, el 87\% de las tareas del hogar son realizadas por las mujeres, frente a sólo el $45 \%$ en las que se involucra el hombre. Deaux y Lewis (1984, citado en Zaro, 1999) estudiaron los estereotipos alusivos a las profesiones y atribuyeron oficios asistenciales a las mujeres, como enfermeras o maestras, apartándoles asi, de profesiones que implican dirección y/o responsabilidad

Precisamente, el ámbito laboral es uno de los más afectados por la desigualdad de género. Las mujeres reciben sueldos más bajos, tienen puestos de trabajo más precarios y mayor inestabilidad y discriminación, tienen más frecuencia de reducciones de jornada para el cuidado de otras personas y ello repercute económicamente en sus recursos, en muchos casos también tienen menos propiedades y bienes. Pese a estar igualmente cualificadas, es muchísimo menor el porcentaje de mujeres que acceden a altos cargos y puestos de mando. Esto haría referencia al denominado techo de cristal, todas aquellas barreras invisibles que provocan la dificultaban la incorporación de la mujer al mundo laboral. El techo de cristal, está presente en otras áreas, como el mundo de la literatura, las artes, la ciencia y el descubrimiento, donde siguen siendo los hombres los que más reconocimientos tienen (Guil Bozal, 2008).

\section{Socialización del género}

El género es el resultado de una cultura y un contexto social del individuo (Yubero Jiménez y Navarro Olivas, 2017). La socialización es un proceso que nos acompaña durante toda la vida y que no se produce de forma uniforme en todas las sociedades y culturas, ni en cada época histórica (Moreno y Ruiz, 2026). En este proceso, intervienen principalmente la familia, la escuela, medios de comunicación y grupos de iguales, transmisores todos ellos de diferentes mensajes, ideas o intereses, acerca de cómo debe ser el comportamiento ideal tanto en una mujer como en un hombre. Por lo tanto, se entiende como socialización del género el proceso por el cual las personas nos convertimos en mujeres u hombres en un momento de una época determinada. En estos procesos de socialización, tienen un papel importante los procesos socio-estructurales, es decir, la persistente utilización del sistema patriarcal donde existe la división de trabajo entre hombres y mujeres. También nos encontramos con los procesos socio-interactivos, donde las oportunidades que se le ofrecen a las mujeres y a los hombres son diferentes, lo que generará en la sociedad una actitud diferente en ambos sexos tras vivir diferentes experiencias durante sus vidas. Debemos tener en cuenta que la socialización facilita la aceptación de diferentes valores, comportamientos y relaciones que existen en la sociedad, lo que permitirá un mayor intercambio de opiniones y con ello, una brecha de oportunidades 
para entender mejor las desigualdades y diferencias. La socialización posibilita la incorporación de los valores predominantes en la sociedad y permite a su vez, el entendimiento y adaptación a los nuevos cambios, siempre de acuerdo con el contexto social al que está ligada.

\section{Justificación}

Realizar una investigación sobre los estereotipos de género en la infancia es fundamental para poder observar y corregir a tiempo todo aquello que influye en el desarrollo personal del niño o niña. Es necesario comenzar a tratar la igualdad de género y a su vez, evitar desde un primer momento los micro-machismos que niños y niñas comienzan a interiorizar desde una edad temprana. Es en la escuela, principalmente en la etapa de pre-escolar cuando los más pequeños comienzan a actuar de una forma u otra, condicionados principalmente por los influyentes de su entorno.

Pese a la importancia que tiene realizar educación en igualdad, lo cierto es, que hasta la fecha no son tan abundantes los estudios interesados en la evaluación de estereotipos de género ya desde la primera infancia. Algo similar ocurre en el periodo adolescente, en la que este tipo de contenidos apenas se trabajan y cuando se hace, en ocasiones se hace de forma inconexa y descontextualizada (Usategui y del Valle, 2007). Además, en numerosas ocasiones el profesorado ha confundido coeducación con escuela mixta y no ha sabido de-construir y reelaborar el modelo de masculinidad imperante, tampoco favorecer la autoestima y autonomía de las mujeres ni revalorizar sus aportaciones.

Tenida en cuenta que somos una sociedad en continuo cambio y desarrollo, se hace imprescindible tener en consideración este tipo de cuestiones que condicionan considerablemente las relaciones entre géneros, distorsionando la realidad y marcando y controlando las pautas de comportamiento socialmente esperadas. Evaluar desde edades tempranas los estereotipos de género nos permite reflexionar acerca de cómo construimos nuestra sociedad y qué valores estamos transmitiendo a nuestros/as niños/as. Dado que ellos constituyen la sociedad del futuro, su educación en igualdad es una cuestión primordial.

\section{MARCO EMPIRICO}

\section{Objetivos}

El objetivo general es comprobar y analizar a través de un cuestionario realizado sobre "el aspecto físico, juguetes y profesiones", a niños de edades comprendidas entre 4 y 5 años, como los estereotipos influyen en ellos desde una edad temprana.

Los objetivos específicos son:

Comprobar si variables como el nivel socio-cultural de los padres influye en el niño/a con la presencia de estereotipos.

Encontrar patrones diferenciales de respuesta en función del sexo.

\section{Hipótesis}

Nuestra hipótesis de partida, dada la literatura revisada es que ya se encontrarán estereotipos de género a estas edades comprendidas entre los 4 y 5 años. Esperamos, por tanto, encontrar patrones diferenciales de respuesta en función del sexo de los participantes, tanto en lo relativo al "aspecto físico, juguetes y profesiones".

\section{Participantes}

\section{Metodología}

La muestra del estudio quedó conformada por 8 niños y 10 niñas de 4 y 5 años $(X=4.75$; DT $=0.31)$. Todos ellos cursaban $2^{\circ}$ de infantil y procedían de un nivel socioeconómico medio. El muestreo fue no aleatorio incidental, aprovechando el acceso a estos participantes durante el periodo de prácticas curriculares.
Tabla 1

Datos sociodemográficos participantes

\begin{tabular}{lccc}
\hline & N & Edad X & DT \\
\hline Niños & $8(44.4 \%)$ & 4.75 & 0.29 \\
Niñas & $10(55.5 \%)$ & 4.75 & 0.33 \\
Total & 18 & 4.75 & 0.31 \\
\hline
\end{tabular}

\section{Instrumentos}

-Cuestionario (ver anexo 2). Se trata de un cuestionario que consta de 12 preguntas organizadas en tres categorías: categoría aspecto físico ( 2 ítems), categoría juguetes (5 ítems) y categoría profesiones (4 ítems), así como una pregunta abierta "¿Qué te gustaría ser de mayor?" en las que se evalúan estereotipos asociados tradicionalmente al género. Por citar algunos ejemplos, se evalúan estereotipos del tipo "el rosa es de chicas", "el balón es un juguete de chicos", "o la profesión de bombero es de chicos". Dicho cuestionario fue diseñado específicamente para este estudio, tras revisar la literatura disponible relacionada con la temática.

-Tarjetas de juguetes y profesiones. Se trata de 11 tarjetas impresas en cartulina tamaño $10 \times 2 \mathrm{cms}$ con imágenes referentes a las diversas categorías. En la tarjeta que aludía al aspecto físico aparecía una muñeca sin ropa (tapando sus zonas íntimas), una camisa de color azul claro y una camisa de color rosa. Las imágenes que mostraban los juguetes mostraban juegos reales: balón de futbol de color blanco y rojo, varios puzles con tonos azulados y una cuna y una muñeca en tonos rosados. Se eligieron estos juguetes ya que son los más usuales en el juego de los niños y niñas de infantil. Respecto a las tarjetas de profesiones, aparecían dos bomberos, dos cocineros, dos policías y dos personas dedicadas al servicio de limpieza. Dos tarjetas que han pertenecido a la categoría de profesiones también, son las imágenes de un juego de médico, donde aparecían las herramientas que se utilizan para dicha profesión (partes de ellas) y un juego de policía, mostrando una pistola y placa de juguete. Para no sesgar las respuestas de los/as niños/ as, en cada tarjeta aparecía siempre un hombre y una mujer desempeñando cada profesión.

\section{Procedimiento}

En primer lugar, se contactó con el centro educativo, y se explicó el procedimiento a la dirección del centro. Posteriormente, tras conseguir su aprobación, decidió trabajarse con el aula de 4 años por razones de conveniencia para la investigadora que realizaba las prácticas académicas en dicha aula. A continuación, tras una revisión exhaustiva de la literatura disponible, y las orientaciones de las directoras como juicio de expertos, se elaboró un breve cuestionario que contiene tanto preguntas al uso, como preguntas apoyadas en fotografías (¿por ej. "este juguete, es para niños, para niñas o para los dos?") (ver anexo 2). Una vez elaborado el cuestionario, se solicitó el consentimiento informado a los padres de los participantes (ver anexo 3). Tras obtenerlo, se evaluó de forma individual a cada uno de los participantes en sesiones de aproximadamente 10 minutos de duración.

El estudio se llevó a cabo en el transcurso de 2 semanas de sesiones de duración. Dicha evaluación se hizo en un rincón dentro del aula en un contexto distendido y libre de distractores. Los resultados se anotaron en la hoja de registro (ver anexo 1) y finalmente, se analizaron los resultados, así como las implicaciones prácticas. 


\section{Análisis de datos}

\section{Resultados}

En primer lugar, con respecto a la pregunta ¿Qué te gustaría ser de mayor? se observan patrones diferenciales en función del sexo (ver tabla 1). En el caso de las niñas, la profesión más seleccionada es peluquera $(n=3)$, seguida de maestra y policía $(n=2)$. En el caso de los niños, policía, seguido de futbolista y constructor $(\mathrm{n}=2)$ (tabla 2$)$.

Tabla2

Descriptivos sobre elección de profesión

\begin{tabular}{lcll}
\hline Respuesta niñas & $\mathbf{n}$ & Respuesta niños & $\mathbf{n}$ \\
\hline Estilista & 1 & Cocinero & 1 \\
Bombera & 1 & Futbolista & 2 \\
Maestra & 2 & Constructor & 2 \\
Peluquera & 3 & Bombero & 1 \\
Policía & 2 & Policia & 3 \\
\hline
\end{tabular}

Para el análisis de los datos se agruparon las preguntas atendiendo a las categorías aspecto físico, juguetes y profesiones y se calcularon los porcentajes de respuesta para cada uno. En primer lugar, se presentan los porcentajes totales, y posteriormente, desglosados por sexos.

En lo referente a la apariencia física (¿con qué camisa vestirías a la muñeca?), tomando los datos totales, un $83.88 \%$ eligen la camisa rosa, siendo solamente un $16.66 \%$ el porcentaje total de niños/as los que han elegido la camisa azul.

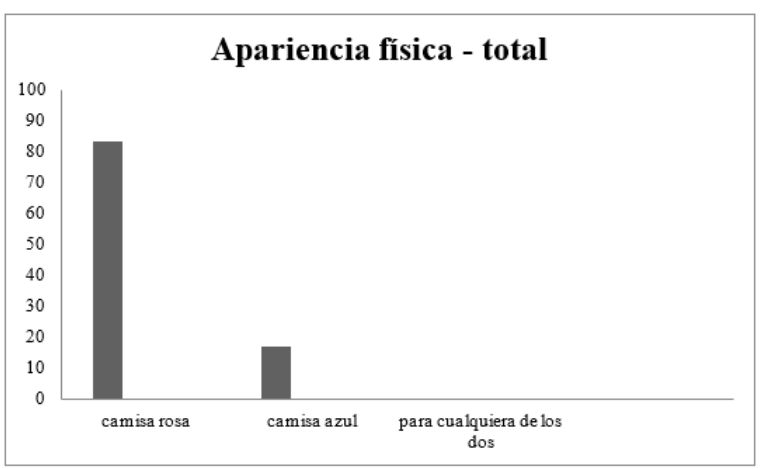

Figura 1. Pregunta 2. \% de respuestas totales de niños y niñas para la elección de cada camisa. ¿Con qué camisa vestirías a la muñeca?

Al hacer la comparativa por sexos, encontramos que el $87 \%$ de los niños eligen la camisa rosa y el $80 \%$ de las niñas, el rosa. (figuras 2 y 3).

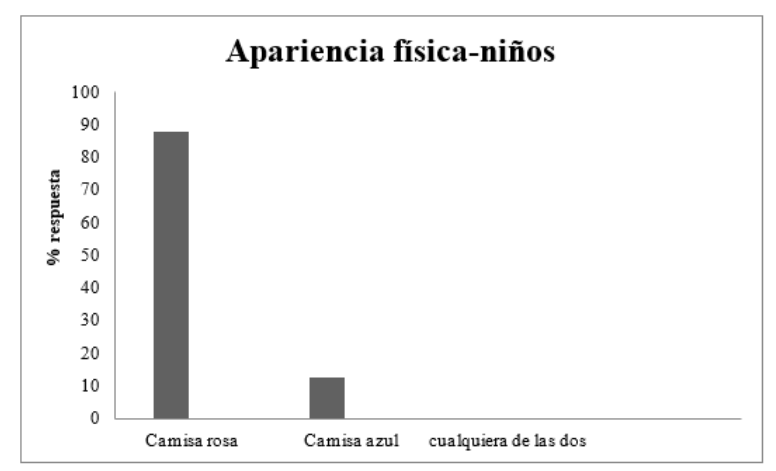

Figura 2. Pregunta 2.\% de respuestas por sexo ¿Con qué camisa vestirías a la muñeca?

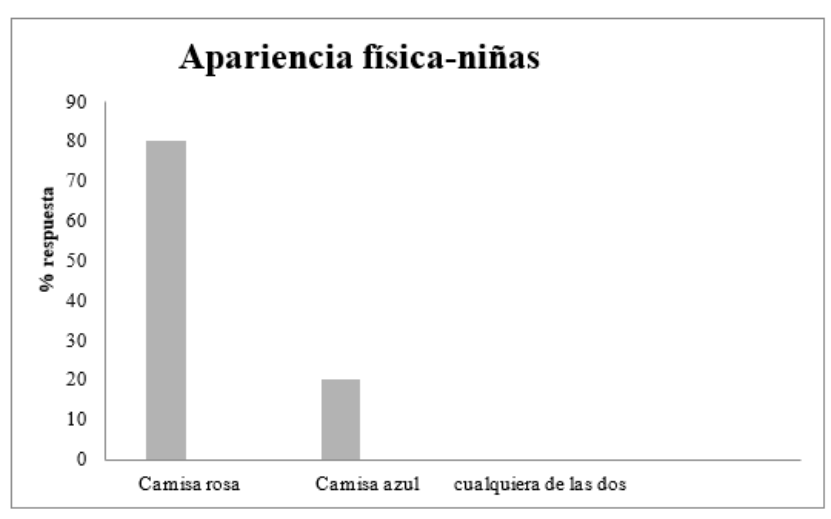

Figura 3. Pregunta 2. \% de respuestas por sexo ¿Con qué camisa vestirías a la muñeca?

Con respecto a la categoría juguetes, analizando el total de la muestra, encontramos que un $88.88 \%$ de los entrevistados sostienen que un balón es para niños, un $100 \%$ coindicen al señalar que la muñeca es para niñas y un $61.11 \%$ apuntan que el puzzle es para niños. La categoría "para ambos" sólo es señalada por un $12.5 \%$ al referirse al balón y un $37.5 \%$ al referirse al puzzle.

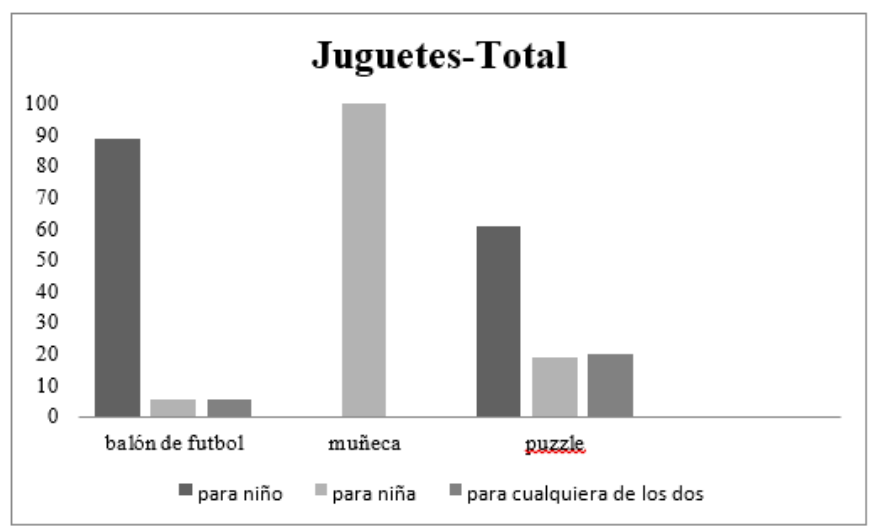

Figura 4. Preguntas 3, 4 y 5. \% de respuestas totales de niñas y niños categoría juguetes

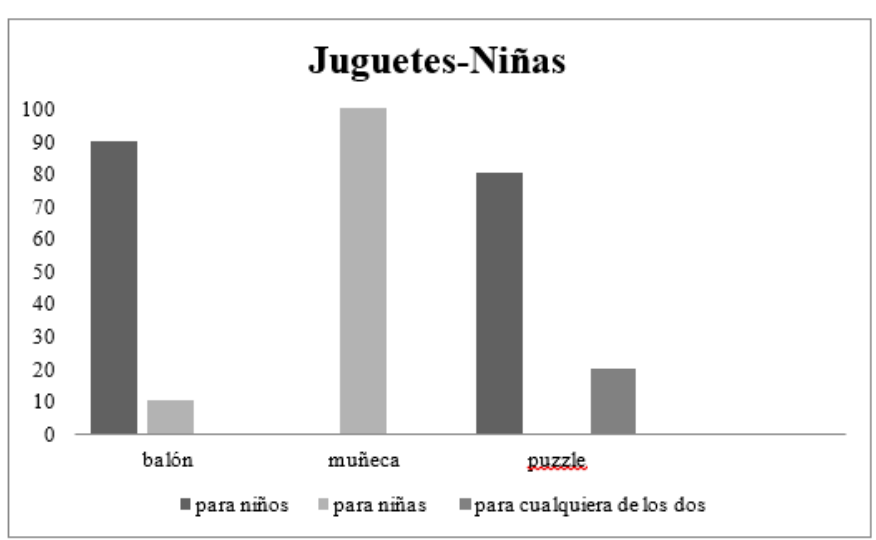

Figura 5. Preguntas 3, 4 y 5. \% de respuestas de niñas en categoría juguetes 
En el caso de los niños, un $87.5 \%$ señala que el balón es un juguete para niños y un $12.5 \%$ para ambos. El $100 \%$ de los niños señala que la muñeca es un juguete para niñas, y en cuanto al puzzle, un $62.5 \%$ señala que es para niños y un $37.5 \%$ que puede ser para ambos.

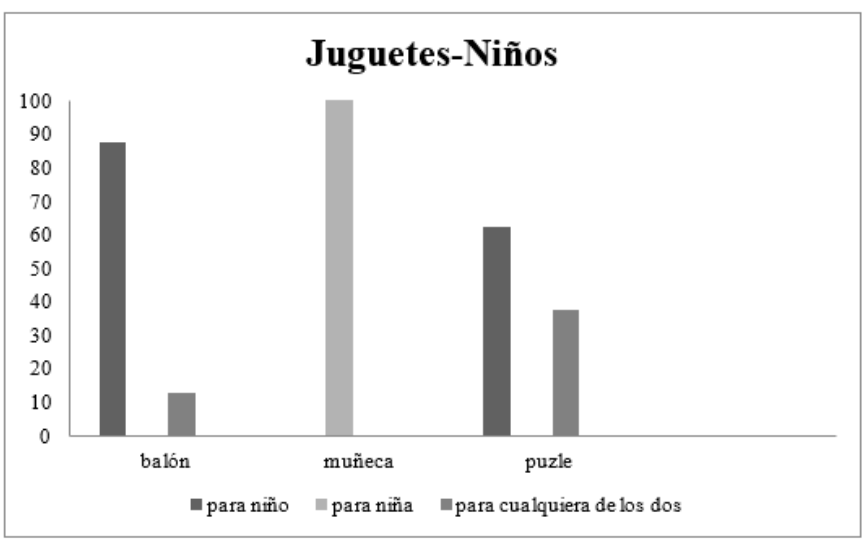

Figura 6. Preguntas 3, 4 y 5. \% de respuestas de niños en categoría juguetes

En lo relativo a la categoría profesiones, analizando el porcentaje total de la muestra, encontramos que un $61.11 \%$ de los entrevistados sostienen que el trabajo de limpieza es de niños, frente al $38.88 \%$ que opina que es para niñas. Por otro lado, el trabajo de cocinero ha sido señalado por un $61.11 \%$ como para "niño", por un $33.33 \%$ para niña y sólo un $5.5 \%$ dijeron que ese trabajo podría ser desempeñado por ambos. La profesión de bombero fue señalada como para "niño" por un $88,88 \%$ del total, frente a sólo un $11.11 \%$ que opina que es para niña. El trabajo de policía fue señalado como para "niño" por un $66.66 \%$ de la muestra. Con respecto a la profesión de médico, un $55.5 \%$ de la muestra lo señaló como para niñas, frente al es para niños es de un $27.7 \%$ para niños y el $16.66 \%$ que lo señaló para ambos. Por último, el trabajo de mecánico fue señalado para “niños" por un $94.44 \%$ y sólo un $5.33 \%$ lo señalaron como para "niñas".

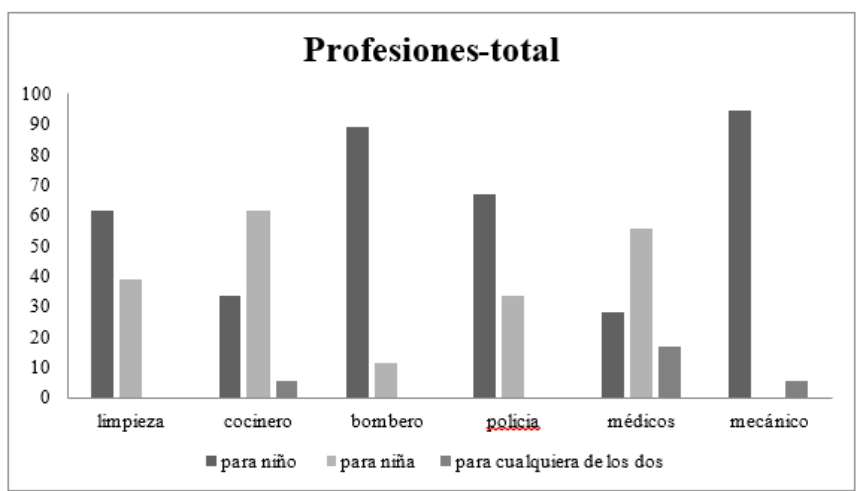

Figura 7. Preguntas 6, 7, 8,9, 10, 11 y 12 . \% total de respuestas niños y niñas categoría profesiones.

Respecto al \% de respuestas obtenidas de las niñas frente a la pregunta de si el trabajo de limpieza es para niño, para niña o para cualquiera de los dos, el \% de respuestas obtenidas es de un 70\% las niñas que decían que era para niño. Del trabajo de cocinero, el $80 \%$ ha coincidido en que era para niña. E1 100\% de las niñas opinan que el trabajo de bombero era para niños. Un $50 \%$ ha señalado que el trabajo de policía era para niño. Respecto al trabajo de médico, un $50 \%$ ha señalado que era para niñas y solamente un $20 \%$ para cualquiera de los dos. Por último, un $90 \%$ señalado que el trabajo de mecánico era para niños y un $10 \%$ para ambos.

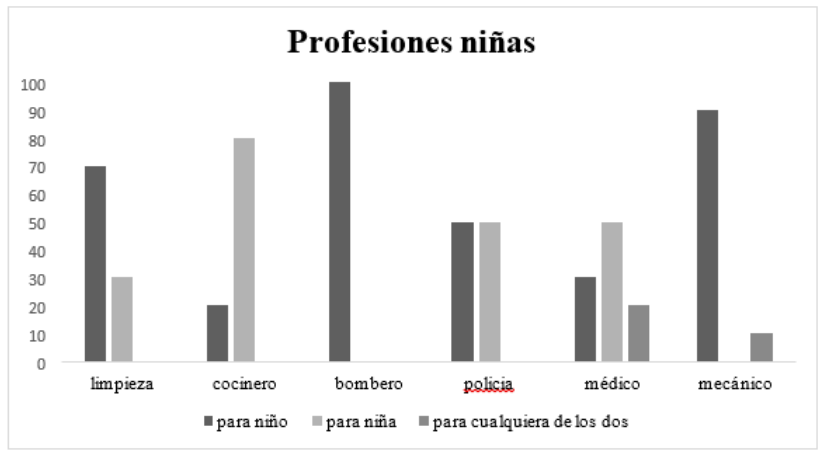

Figura 8. Preguntas 6, 7, 8,9, 10, 11 y 12.\% de respuestas niñas categoría profesiones

En la categoría de profesiones referente a los resultados de los niños, observamos que el \% de niños que señalan el trabajo de limpieza como profesión para niño es de un $50 \%$, siendo el resto el mismo \% que opina que es para niña. Un 50\% indica como trabajo de niño, la profesión de cocinero, mientras que un $12.5 \%$ lo considera para ambos. Un $75 \%$ coincide en que piensan que el trabajo de bombero es para niño. El trabajo como policía obtiene un $100 \%$ total de respuestas en las que el alumnado coincide en que es para niño, al igual que el trabajo como mecánico, donde el 100\% de los niños coinciden en considerarlo como una profesión de niños. En el caso de la profesión de médico, los niños coinciden en la mayoría con un $62.5 \%$ de respuestas en que es un trabajo para "niñas", quedando un $12.5 \%$ restante que piensa que es para ambos.

\section{Profesiones niños}

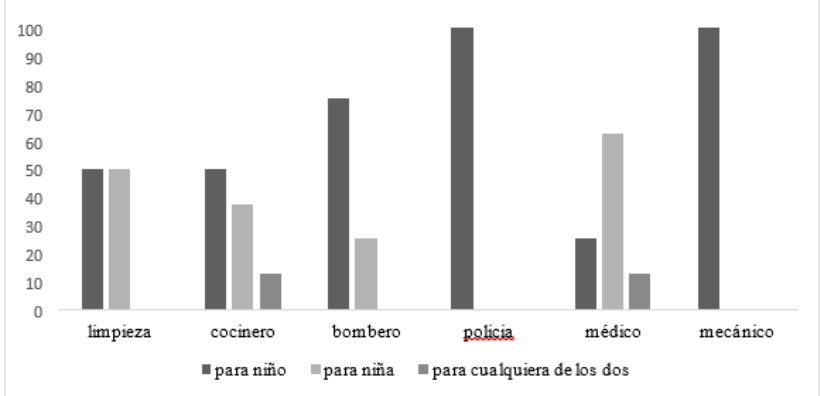

Figura 9. Preguntas 6, 7, 8,9, 10, 11 y $12 . \%$ de respuestas niños categoría profesiones.

En la categoría de profesiones referente a los resultados de los niños, observamos que el \% de niños que señalan el trabajo de limpieza como profesión para niño es de un 50\%, siendo el resto el mismo \% que opina que es para niña. Un 50\% indica como trabajo de niño, la profesión de cocinero, mientras que un $12.5 \%$ lo considera para ambos. Un $75 \%$ coincide en que piensan que el trabajo de bombero es para niño. El trabajo como policía obtiene un $100 \%$ total de respuestas en las que el alumnado coincide en que es para niño, al igual que el trabajo como mecánico, donde el $100 \%$ de los niños coinciden en considerarlo como una profesión de niños. En el caso de la profesión de médico, los 
niños coinciden en la mayoría con un $62.5 \%$ de respuestas en que es un trabajo para "niñas", quedando un $12.5 \%$ restante que piensa que es para ambos.

\section{Discusión}

El objetivo del presente estudio era obtener un perfil de los estereotipos de género en niños y niñas de edad infantil. Del análisis de las respuestas de estos preescolares a un breve cuestionario, pueden extraerse interesantes conclusiones.

En primer lugar, la pregunta, ¿qué quieres ser de mayor?, arroja respuestas diferenciales en función del sexo. En el caso de los niños, las profesiones de futbolista y constructor son las más elegidas, mientras que en el caso de las niñas, peluquera y maestra. Pero ha sido la profesión de policía en la que más han coincidido el número de niños y de niñas en elegirla. Se trata de un dato bastante interesante ya que el número de policías masculinos en España es mucho mayor al de mujeres. Según un estudio del Plan operativo de igualdad de la policía en Madrid (2018), la presencia de la mujer en la Policía Local es de un $12.32 \%$ frente a un $87.68 \%$ en hombres. En cualquier caso, parece que se perpetúan los estereotipos establecidos: las mujeres, están más orientadas a las profesiones destinadas a la imagen física, o a la enseñanza y al cuidado como son los trabajos de estilista, peluquera o maestra mientras que los hombres se ocupan en 'profesiones destinadas a ejercer control, mantener el orden y ofrecer protección, como son los trabajos de bombero y policía.

Por otro lado, en el área de apariencia física, parece mantenerse la típica asociación culturalmente establecida: rosa para chicas y azul para chicos. De hecho, esa fue la respuesta que aquellos/as que eligieron rosa para la muñeca daban cuando se les pedía que justificasen su respuesta ("porque el color rosa es de niña"). Ya desde una edad temprana se observa la notable influencia de nuestra sociedad, que se ve reforzada por el grupo de iguales. Por el contrario, el número de niños y niñas que eligieron la camisa de color azul es mínimo y justificaron su respuesta aludiendo a gustos personales "el azul también es para niña y a mí me gusta". Esto podría sugerirnos también diferencias con respecto a su educación en el entorno más próximo. Esta asignación de colores es totalmente arbitraria y promovida culturalmente. De hecho, en el siglo XIX, la regla generalmente aceptada era rosa para los chicos y azul para las chicas (Girela, 2019).

En lo relativo a la categoría juguetes, se observan también patrones diferenciales. Así, mientras, por un lado, la muñeca es el juguete por antonomasia para niñas, coincidiendo el total de la muestra en esta afirmación, para los niños lo es el balón. Aquí ya se entrevé el fuerte impacto de la cultura en el proceso de socialización. Sin ir más lejos, los medios de comunicación crean un gran impacto en los niños y niñas ya que se trata de grandes influyentes audiovisuales. Según un estudio sobre la publicidad de juguetes en la campaña de Navidad (2006-2007) en el $90.9 \%$ de los casos de anuncios de muñecas aparecen niñas, solas o acompañadas (generalmente de sus madres), mientras que solo en el $6.8 \%$ de ellos aparecen también niños y nunca solos. En el caso de los anuncios dirigidos a los juguetes para niños, en la mayoría de los casos, el balón sale anunciado por uno o varios niños sin presencia del sexo femenino. Respecto a los resultados obtenidos al preguntar por el balón la gran parte de los encuestados coincide al señalar que es un juguete para niña. Por su parte, el puzzle, pese a ser un juguete en apariencia bastante neutro, ha sido señalado por su mayoría como para niños. En este caso, dado que el juguete en sí no está fuertemente asociado en el acervo social a ningún género, se apoyan en elementos extrínsecos al propio juego en sí, como el color de las piezas (en este caso, el puzle, contenía piezas azules, volviendo al estereotipo anterior de "azul es para niños"). Según un estudio sobre la publicidad de juguetes en la campaña de Navidad (2006-2007) en el caso de los anuncios de juguetes con destinatario mixto, como sucede en los anuncios de juegos de mesa como puede ser el puzle, en él juegan niños y niñas, puede asi apreciarse en ocasiones una distribución desigualitaria de roles o actitudes.

En lo que respecta a la categoría de profesiones, se repiten los patrones que se venían observando en los juguetes. En el caso de los niños, estos son identificados con profesiones como bombero, mecánico o policía. Los trabajos en los que los niños y niñas coinciden al señalar como profesión de 'niñas' son los trabajos de cocinero/a y médico.

Ello nos muestra que los niños y niñas son influenciados por lo que refleja la sociedad, es decir, una sociedad en la que esas funciones la ejercen los hombres. Según la Asociación

'Ser bombera' (2018), en nuestro país solo 168 personas de 20000 bomberos, son mujeres. Sin ir más lejos, según el Instituto Nacional de Estadística (2017), muestra una comparativa en el número que hay de médicos en nuestro país, tanto de mujeres como de hombres. En dicho estudio, muestra que había 2.162 mujeres más que hombres en el servicio de sanidad, siendo un total de 125.817 hombres y 127.979 mujeres. Ello puede ser uno de los tantos motivos por el cual los niños y niñas creen que el trabajo de médico es de niñas, pues han podido tener más posibilidades de haber visto a una médica cuando han tenido que ir al pediatra

Es conveniente, no obstante señalar las limitaciones del presente estudio, entre ellas principalmente el bajo tamaño muestral o la homogeneidad de la muestra. Sería interesante replicar estos resultados en una muestra más amplia y observar si se mantienen estas tendencias. Asimismo, todos los alumnos pertenecen a un nivel socioeconómico y educativo muy similar (nivel medio), por lo que sería conveniente explorar qué resultados se obtienen en otros contextos. En cualquier caso, los resultados de esta primera aproximación apuntan a la existencia ya a estas edades tan tempranas de ciertos estereotipos de género. Ante panorama es necesario reflexionar y actuar de forma responsable, sin perder de vista nunca la importancia de nuestra actitud y nuestros valores a la hora de trabajar el desarrollo de los estereotipos. Somos nosotros quienes generamos expectativos que posteriormente reflejamos en nuestros niños y niñas. En lo referido a los maestros y maestras, asumimos en colaboración con los progenitores un papel fundamental en el proceso de educación en valores. Afortunadamente, en las últimas décadas, estamos asistiendo a cambios en los roles asignados tradicionalmente a las mujeres y los hombres, en aras a la igualdad (las mujeres han ido incorporándose al trabajo remunerado, la educación, progresivamente encontramos más mujeres que ocupan cargos de responsabilidad en empresas y hombres que se dedican a las responsabilidades del cuidado doméstico). No obstante, estos cambios aún siguen siendo cambios lentos y los roles tradicionales siguen perviviendo con los que intentan actualizarse a los tiempos actuales. Es tarea nuestra y responsabilidad social trabajar para educar a nuestros niños y niñas desde un plano de igualdad que evite la perpetuación de estereotipos de género y que garantice las mismas oportunidades a niños y niñas.

\section{Referencias}

Barberá Heredia, E., Ramos A. y Candela, C. (2000). Más allá del "techo de cristal". Diversidad de género. Revista del Ministerio de Trabajo y Asuntos Sociales, 40, 5568.

BBC. (2017). Los 10 peores países para ir a la escuela si eres niña. Recuperado el 20-3-20, de BBC EL MUNDO. https://www.bbc.com/mundo/ noticias 41588118.

Bosada, M. (2018). ¿Cómo trabajar la igualdad de género en el colegio desde la infancia? Recuperado el 8-3-2020 de: https:// www.educaweb.com/noticia/2018/06/26/propuestas-educar- 
igualdadgenero-educacion-infantil-primaria-18509/

Bourque, S., Butler, J. y Lamas, M. (1996). El Género. México: Universidad Nacional Autónoma de México.

Campus training (2018). Mujer policía: porcentaje en los Cuerpos y Fuerzas de Seguridad. Recuperado el 13-3-2020 de: https:// www.campustraining.es/noticias/mujerpolicia/

Chinchilla, N. Poelmans, S y León, C. (2005). Mujeres directivas bajo el techo de cristal. Recuperado de: https://scholar.google.com.uy/ scholar?hl $=$ es\&as_sdt $=0 \% 2 \mathrm{C} 5 \& \mathrm{q}=$ Chinchilla $\% 2 \mathrm{C}+\mathrm{N} .+$ Poelmans $\%$ $2 \mathrm{C}+\mathrm{S}+\mathrm{y}+\mathrm{Le} \% \mathrm{C} 3 \% \mathrm{~B} 3 \mathrm{n} \% 2 \mathrm{C}+\mathrm{C} .+\% 282005 \% 29 .+\& b \operatorname{tnG}=$

Con salud (2018). El número de médicas colegiadas supera por primera vez al de médicos. Recuperado de: https://www.consalud.es/profesionales/ el-numero-de-medicascolegiadas-supera-por-primera-vez-en-la-historia -al-de medicos_55524_102.html

Conway, J., Bourque, S. y Scott, J. (1996). El concepto de género. En: M. Lamas (comp.), El género. La construcción cultural de la diferencia sexual (pp. 21-33). México: PUEG-UNAM.

Erikson, E. H. (1974). Identidad, juventud y crisis. Buenos Aires: Paidós.

Espinosa-Bayal, (2006). La construcción del género desde el ámbito educativo. Comunicar, 2-8.

Feijoo, A, (2015). estereotipos en los disfraces de carnaval. Recuperado de: https://www.realkiddys.com/estereotipos-en-los-disfraces-carnaval/ Gallegos Argüello, M. C. (2012). La identidad de género: masculino versus femenino. Trabajo presentado en I Congreso Internacional de Comunicación y Género, 705718.

Gómez. M.V. (2018). Una mujer gana un 13\% menos que un hombre en trabajos similares. Recuperado de: https://elpais.com/ economia/2018/03/06/actualidad/1520362982_401961.html

González Gavaldón, B. (1999). Los estereotipos como factor de socialización en el género. Comunicar, 12, 79-88.

Hernández Morales, G. y Jaramillo Guijarro, C. (2003). La educación sexual en la primera infancia. Guías para madres, padres y profesorado de Educación Infantil: Ministerio de Educación. Madrid, España.

Kohlberg, L. (1969).La educación moral. Recuperado de: https:// www.redalyc.org/pdf/2090/209014646001.pdf

Lumbreras-Castellanos, O. (2013). Estereotipos de género en los juguetes de los niños. Logroño, España: Browne.

Martinez, J. (2018) El juego es cosa seria. Los juguetes y los estereotipos de género: MaguaRED.. Recuperado de: https://maguared.gov.co/losjuguetes-y-losestereotipos-de-genero/

Moreno, M. y Ruiz, C. (2016). Cambios sociales y género. Madrid: Anaya.

Parra, N. (2018). Sexualidad. Cuerpos, identidades, y orientaciones. Canarias: Instituto Canario de Igualdad. Gobierno de Canarias.

Save the children, (2016). Todo lo que sabemos sobre los géneros es relativo: el rosa era un color de chicos hace 80 años. Recuperado de: https:// magnet.xataka.com/endiez-minutos/el-rosa-era-el-color-de-los-chicoshace-80-anos-y-otras-historias-desexismo-infantil

Serbombera, (2018). En España hay alrededor de 2000 bomberos, sólo 168 son mujeres. Recuperado de: https://www.serviciosemergencia.es/ noticia/1462/reportaje-enespana-hay-alrededor-de-20-000-bomberossolo-168-son-mujeres

Shaffer, D. R. (2000). Psicología del desarrollo: infancia y adolescencia. México: S. A. Ediciones paraninfo.

Silvestre Cabreara, M. (2010). Masculinidades e igualdad: análisis multidisciplinar. Recuperado de: https://www.emakunde.euskadi.eus/ contenidos/informacion/gizonduz_dokumentu ak/es_def/adjuntos/ masculinidades_e_igualdad.pdf.

Usategi, E. y Del Valle, A. I. (2012). Aprender a formar. Valores en la formación inicial del profesorado. Vitoria-Gasteiz: Fundación Fernando Buesa.

Unger, G. F. (1989). Formación y desarrollo de la identidad sexual en la infancia y adolescencia. Nueva York: Baywood.

Vazquez, L. (2020). Estereotipos de género: los juguetes. Recuperado de: https://www.vix.com/es/imj/familia/5092/estereotipos-degenero-los-juguetes.

Yubero Jiménez, S. y Navarro Olivas, R. (2017). Socialización del género. Recuperado de https://www.researchgate.net/ publication/280157550_Socializacion_de_genero 